\title{
Os Hesperiidae (Lepidoptera, Hesperioidea) da RPPN Klagesi, Santo Antônio do Tauá, Pará, Brasil: nova contribuição para o conhecimento da biodiversidade da área de endemismo Belém
}

\author{
Olaf H. H. MIELKE ${ }^{1}$, Eduardo CARNEIRO ${ }^{1}$, Mirna M. CASAGRANDE ${ }^{1}$
}

RESUMO

Embora a região Belém seja uma das áreas mais exploradas da Amazônia com coletas desde o século XVIII, grande parte das informaçóes se encontra dispersa em coleçóes internacionais, o que dificulta a reunião de dados e interpretaçóes biogeográficas. Visando ampliar o conhecimento da fauna de lepidópteros da região, o presente estudo levantou uma lista de 225 espécies de Hesperiidae encontradas na RPPN Klagesi durante aproximadamente 24 anos de amostragens esporádicas, embora mais freqüentemente na última década. São adicionadas 113 espécies previamente não relacionadas para a região, a qual passa a ser a área de maior riqueza conhecida de Hesperiidae dentre todas as regióes de endemismo da Amazônia brasileira até então inventariadas. No entanto, o conhecimento da diversidade de Hesperiidae em outras áreas de endemismo é tão restrito, que a riqueza de espécies deve ser utilizada apenas como indicador de esforço amostral ao invés de inferências biogeográficas. Três das regióes de endemismo ainda não apresentam nenhum inventário de fauna (Xingú, Napo e Imeri), enquanto as demais são reconhecidas por um ou poucos inventários. Portanto, para que a lepidopterofauna amazônica seja realmente conhecida e utilizada em estudos de biogeografia e conservação, são necessários esforços direcionados nestas regiôes ainda totalmente desconhecidas ou mesmo a reunião de informaçóes sobre coletas já realizadas, cujo material se encontra disperso em coleçóes pelo mundo.

PALAVRAS-CHAVE: inventário, distribuição, borboletas, Floresta Amazônica

\section{The Hesperiidae (Lepidoptera, Hesperioidea) from RPPN Klagesi, Santo Antônio do Tauá, Pará, Brazil: a new contribution to biodiversity's knowledge of Belém endemism area}

\begin{abstract}
The region of Belém is one of the best known area of the Amazon Forest, given its scientific explorations since the $18^{\text {th }}$ century. However much of the informations about the biological diversity of the region is scattered at several museums around the world, thus making it harder to access and to propose biogeographical hypothesis.. Aiming to contribute to the knowledge of lepidopterological diversity of the region, the present study lists 225 species of Hesperiidae present at Private Natural Reserve Klagesi registered during approximately 24 years of sporadically collecting, although more frequently on the past decade. One hundred and thirteen species not yet registered in endemism's region of Belém are added, which makes it the richest of all Amazonian endemism areas in Brazil. However, the Amazonian skippers are so poorly known that this richness index can only be used for effort comparisons instead of biogeographical or ecological inferences. Three of the endemism regions still lack information about their diversity (Xingú, Napo and Imeri), while others are known basically from one or a few inventories. Therefore, focused sampling at those unknown areas or gathering information at international museums are indispensable strategies to obtain a better knowledge of Amazonian butterfly diversity so they can be used for biogeographical studies and conservation management.
\end{abstract}

KEYWORDS: inventory, distribution, skippers, Amazon Forest

1 Laboratório de Estudos de Lepidoptera Neotropical, Departamento de Zoologia, UFPR. Caixa Postal 19020, 81531-980 Curitiba, Paraná, Brasil. omhesp@ufpr.br 


\section{INTRODUÇÃO}

As justificativas para a realizaçáo de inventários de lepidopterofauna no Brasil se direcionam basicamente para a ausência de informaçóes sobre a biodiversidade local e/ou escassez de amostragens direcionadas ao grupo estudado. De fato, mesmo com amplas regióes no Brasil ainda são desconhecidas (Santos et al. 2008), a região de Belém certamente não se enquadra nesta categoria. Por oferecer a entrada para a região Amazônica e ser de fácil acesso, muitos naturalistas utilizavam a cidade de Belém (geralmente mencionada em estudos antigos simplesmente como Pará) como principal ponto para coletas de borboletas e envio de material para a Europa (Wallace 1853; Bates 1989). Assim, uma quantidade significativa de informaçóes sobre a biodiversidade de diversos grupos de borboletas na região se encontra dispersa em diferentes museus e coleçôes brasileiras e estrangeiras, sendo que apenas duas listas foram publicadas para esta regiáo até o momento (Stichel 1923; Mielke 1973). Para Hesperiidae em especial, a única lista disponível (Mielke 1973) acumula significativo esforço amostral (43 dias de coleta), embora concentrado em uma única estação do ano.

Embora a floresta amazônica, como um todo, ainda não se mostre significativamente ameaçada por pressōes antrópicas, algumas de suas áreas de endemismo (sensu Cracraft 1985 e Silva et al. 2002) já apresentam índices de desmatamento significativo (Gascon et al. 2001). A regiáo de endemismo de Belém, em especial, além de ter tido cerca de dois terços de sua área desmatada, apresenta também a menor totalidade de áreas protegidas (Silva et al. 2005), contribuindo para que seja considerada a área de endemismo de maior ameaça para a biodiversidade da Amazônia.

Sendo assim, embora a regiáo de Belém ter sido bem amostrada desde o século XVIII, ainda faltam estudos que reunam tais informaçóes para melhor descrever a região e respectiva fauna, a qual nos dias atuais se encontra sobre forte interferência humana. Desta forma, o presente estudo apresenta uma lista de espécies de Hesperiidae (Lepidoptera, Hesperioidea) da Reserva Particular do Patrimônio Natural (RPPN) Klagesi, uma reserva localizada no município de Santo Antônio de Tauá, nos arredores de Belém, objetivando contribuir para o conhecimento da fauna da área de endemismo Belém e acrescer dados sobre a biodiversidade amazônica.

\section{MATERIAL E MÉTODOS}

A RPPN Klagesi (-1.1663 S e -48.1296 W) se localiza no município de Santo Antônio do Tauá, Pará, a 40m de altitude. Constitui atualmente um fragmento de 23 ha de floresta ombrófila densa de terra firme com pouca declividade. O clima é do tipo Af - tropical úmido sem estação seca (classificação de Köppen-Geiger), com temperatura média anual em torno de $26^{\circ} \mathrm{C}$ e precipitação variando de 2500 a $3000 \mathrm{~mm}$.

A presente lista foi obtida através de coletas aleatórias realizadas na RPPN Klagesi pelo proprietário e pesquisador autônomo Pierre Jauffrett, o qual amostrou intensamente a reserva entre os anos de 1985 e 2009 (mais frequentemente na última década), compreendendo todas as estaçóes do ano. A nomenclatura e classificação das espécies seguiram Mielke (2004; 2005). Todos os espécimes aqui relacionados se encontram tombados na coleçấo entomológica Pe. Jesus Santiago Moure situada no Departamento de Zoologia da Universidade Federal do Paraná.

\section{RESULTADOS E DISCUSSÃO}

Foram relacionadas, ao total, 225 espécies de Hesperiidae encontradas na RPPN Klagesi, dentre elas: 7 Pyrrhopyginae, 116 Pyrginae e 102 Hesperiinae, ultrapassado o número de espécies relacionadas por Mielke (1973) para outras localidades de Belém (Utinga) e arredores (Capim, Ourém e Viseu). É importante ressaltar que o esforço amostral empregado nessas regiôes é também menor e que, portanto, náo é adequado comparar diretamente a riqueza e composiçấo entre as localidades. Para a regiáo de endemismo Belém, o presente estudo adiciona 113 espécies à lista disponibilizada por Mielke (1973), totalizando 272 espécies (Tabela 1). Sendo assim, a regiáo se torna, atualmente, a mais rica em número de espécies de Hesperiidae até entáo coligidas entre as áreas de endemismo amazônicas brasileiras (Tabela 2).

A riqueza entre as áreas de endemismo não foi aqui utilizada para comparaçóes ecológicas ou biogeográficas, mas sim para enfatizar o baixo esforço amostral empregado em cada uma delas. Mielke (1973) estimou que na região das Guianas pudessem existir aproximadamente 400 espécies de Hesperiidae, valor ultrapassado na área de endemismo Inambari no Peru (411 spp. coligidas na Reserva de Tambopata e Parque Nacional Del Manu, Madre de Dios) (Lamas 1981; Lamas 1985; Robbins et al. 1996). No entanto, no Brasil, nenhuma área de endemismo sequer se aproxima desta riqueza, devido principalmente ao baixo esforço amostral empregado em levantamentos amazônicos (Santos et al. 2008). Em contrapartida, observa-se nas localidades peruanas um intenso esforço amostral realizado durante mais de um ano de coleta em cada um dos estudos. A ausência de inventários no Brasil para três áreas de endemismo brasileiras (Xingu, Napo e Imeri) se deve ainda às restriçóes de acesso a tais localidades, além da falta de incentivos e infra-estrutura local ao pesquisador (Mielke et al. 2011).

Apesar da realização de muitas coletas na regiāo de Belém, a sua fauna, assim como de outras regióes brasileiras, continua mal representada em inventários publicados. Outras regióes amazônicas também foram exploradas sem que apresentassem 
Tabela 1 - Lista de espécies de Hesperiidae da área de endemismo de Belém, distribuídas entre a localidade inventariada (RPPN Klagesi) e demais municípios inventariados por Mielke (1973). Siglas representam: RPPN Klagesi (KL); Parque Utinga, Belém (UT); Município de Ourém (OU); Município de Soure, Illha de Marajó (SO); Município de Capim (CA); e Município de Viseu (VI).

\begin{tabular}{|c|c|c|c|c|c|c|c|}
\hline & & $\mathrm{KL}$ & UT & OU & SO & $\mathrm{CA}$ & VI \\
\hline & Pyrrhopyginae & & & & & & \\
\hline 1 & Elbella azeta azeta (Hewitson, 1866) & $x$ & & & & & \\
\hline 2 & Jemadia fallax fallax (Mabille, 1878) & $x$ & & & & & \\
\hline 3 & $\begin{array}{l}\text { Jemadia hewitsonii hewitsonii } \\
\text { (Mabille, 1878) }\end{array}$ & $x$ & & & & & \\
\hline 4 & Parelbella ahira ahira (Hewitson, 1866) & $x$ & & & & & \\
\hline 5 & $\begin{array}{l}\text { Pyrrhopyge phidias hyperici } \\
\text { Hübner, } 1823\end{array}$ & $x$ & & & & & \\
\hline 6 & Pyrrhopyge proculus lina Bell, 1947 & $x$ & & $x$ & & & \\
\hline \multirow[t]{3}{*}{7} & $\begin{array}{l}\text { Pyrrhopyge thericles orientis } \\
\text { Bell, } 1946\end{array}$ & $x$ & $x$ & & & & \\
\hline & Total & 7 & 1 & 1 & 0 & 0 & 0 \\
\hline & Pyrginae Eudamini & & & & & & \\
\hline 8 & $\begin{array}{l}\text { Aguna aurunce aurunce } \\
\text { (Hewitson, 1867) }\end{array}$ & & $X$ & & & & \\
\hline 9 & Aguna coelus (Stoll, 1781) & $x$ & $x$ & $x$ & & & $x$ \\
\hline 10 & $\begin{array}{l}\text { Astraptes anaphus anaphus } \\
\text { (Cramer, 1777) }\end{array}$ & $x$ & & & & & \\
\hline 11 & $\begin{array}{l}\text { Astraptes apastus apastus } \\
\text { (Cramer, 1777) }\end{array}$ & $\mathrm{X}$ & & & & & \\
\hline 12 & $\begin{array}{l}\text { Astraptes cretatus cretatus } \\
\text { (Hayward, 1939) }\end{array}$ & & & & & $x$ & \\
\hline 13 & $\begin{array}{l}\text { Astraptes creteus creteus } \\
\text { (Cramer, 1780) }\end{array}$ & $x$ & & & & & \\
\hline 14 & Astraptes enotrus (Stoll, 1781) & $X$ & & & & & \\
\hline 15 & $\begin{array}{l}\text { Astraptes fulgerator fulgerator } \\
\text { (Walch, 1775) }\end{array}$ & $x$ & $x$ & $x$ & & $x$ & \\
\hline 16 & Astraptes janeira (Schaus, 1902) & & $X$ & & & & \\
\hline 17 & Astraptes halesius (Hewitson, 1877) & $x$ & & & & & \\
\hline 18 & Astraptes talus (Cramer, 1777) & $x$ & $X$ & & & & \\
\hline 19 & Augiades crinisus (Cramer, 1780) & $x$ & & & $x$ & & \\
\hline 20 & $\begin{array}{l}\text { Augiades epimethea bicolor } \\
\text { (Mabille \& Boullet, 1919) }\end{array}$ & $x$ & & & & & \\
\hline 21 & Autochton itylus Hübner, 1823 & $x$ & $x$ & & & & \\
\hline 22 & Autochton longipennis (Plötz, 1882) & & $x$ & $x$ & & & $x$ \\
\hline 23 & Autochton neis (Geyer, 1832) & $x$ & $X$ & & & $x$ & \\
\hline 24 & Autochton zarex (Hübner, 1818) & $x$ & $x$ & & & & \\
\hline 25 & Bungalotis astylos (Cramer, 1780) & $x$ & & & & & \\
\hline 26 & $\begin{array}{l}\text { Celaenorrhinus syllius } \\
\text { (C. Felder \& R. Felder, 1862) }\end{array}$ & $x$ & & & & & \\
\hline 27 & $\begin{array}{l}\text { Cephise cephise } \\
\text { (Herrich-Schäffer, 1869) }\end{array}$ & $x$ & & $x$ & & & \\
\hline 28 & $\begin{array}{l}\text { Chioides catillus catillus } \\
\text { (Cramer, 1779) }\end{array}$ & $x$ & $X$ & & $x$ & $x$ & \\
\hline 29 & $\begin{array}{l}\text { Chrysoplectrum otriades } \\
\text { (Hewitson, 1867) }\end{array}$ & $x$ & & & & & \\
\hline 30 & $\begin{array}{l}\text { Chrysoplectrum perniciosus } \\
\text { (Herrich-Schäffer, 1869) }\end{array}$ & $x$ & & & & & \\
\hline
\end{tabular}

\begin{tabular}{|c|c|c|c|c|c|c|c|}
\hline & & $\mathrm{KL}$ & UT & OU & SO & $\mathrm{CA}$ & $\mathrm{VI}$ \\
\hline 31 & $\begin{array}{l}\text { Chrysoplectrum pervivax } \\
\text { (Hübner, [1819]) }\end{array}$ & $X$ & & & & & \\
\hline 32 & Drephalys alcmon (Cramer, 1779) & $\mathrm{X}$ & & & & & \\
\hline 33 & Dyscophellus erythras (Mabille, 1888) & $X$ & & & & & \\
\hline 34 & $\begin{array}{l}\text { Dyscophellus ramusis astrapaeus } \\
\text { (Hewitson, 1876) }\end{array}$ & $X$ & & & & & \\
\hline 35 & $\begin{array}{l}\text { Entheus sp. (mencionado como } \\
\text { Entheus gentius) }\end{array}$ & & & & & & $x$ \\
\hline 36 & Entheus telemus Mabille, 1898 & $X$ & $X$ & $\mathrm{X}$ & & $X$ & \\
\hline 37 & $\begin{array}{l}\text { Euriphellus euribates euribates } \\
\text { (Stoll, 1782) }\end{array}$ & $\mathrm{X}$ & & & & & \\
\hline 38 & Hyalothyrus leucomelas (Geyer, 1832) & $X$ & & & & $X$ & \\
\hline 39 & Nascus broteas (Cramer, 1780) & $x$ & & & & & \\
\hline 40 & $\begin{array}{l}\text { Nicephellus nicephorus } \\
\text { (Hewitson, 1876) }\end{array}$ & $X$ & & & & & \\
\hline 41 & Oileides azines (Hewitson, 1867) & $x$ & & & & & \\
\hline 42 & Phanus marshalli (Kirby, 1880) & $X$ & & & & & \\
\hline 43 & Phanus vitreus (Stoll, 1781) & $x$ & $x$ & $x$ & & & \\
\hline 44 & Phareas coeleste Westwood, 1852 & $X$ & & & & & \\
\hline 45 & $\begin{array}{l}\text { Phocides polybius polybius } \\
\text { (Fabricius, 1793) }\end{array}$ & $X$ & & & & & \\
\hline 46 & Polythrix auginus (Hewitson, 1867) & $X$ & & & & & \\
\hline 47 & $\begin{array}{l}\text { Porphyrogenes despecta } \\
\text { (Butler, 1870) }\end{array}$ & $X$ & & & & & \\
\hline 48 & $\begin{array}{l}\text { Porphyrogenes pausias } \\
\text { (Hewitson, 1867) }\end{array}$ & $X$ & & & & & \\
\hline 49 & Salatis salatis (Stoll, 1782) & $x$ & & & & & \\
\hline 50 & Tarsoctenus papias (Hewitson, 1857) & & $x$ & & & & \\
\hline 51 & Typhedanus crameri McHenry, 1960 & $x$ & & & & & \\
\hline 52 & $\begin{array}{l}\text { Typhedanus undulatus } \\
\text { (Hewitson, 1867) }\end{array}$ & $\mathrm{X}$ & & & $X$ & & \\
\hline 53 & Udranomia kikkawai (Weeks, 1906) & $X$ & & & & & \\
\hline 54 & $\begin{array}{l}\text { Udranomia orcinus } \\
\text { (C. Felder \& R. Felder, 1867) }\end{array}$ & $X$ & & & & & \\
\hline 55 & Urbanus belli (Hayward, 1935) & $\mathrm{X}$ & $x$ & $x$ & & & \\
\hline 56 & $\begin{array}{l}\text { Urbanus carmelita carmelita } \\
\text { (Herrich-Schäffer, 1869) }\end{array}$ & & $x$ & & & & \\
\hline 57 & $\begin{array}{l}\text { Urbanus dorantes dorantes } \\
\text { (Stoll, 1790) }\end{array}$ & $X$ & $\mathrm{X}$ & $\mathrm{X}$ & & & $x$ \\
\hline 58 & $\begin{array}{l}\text { Urbanus doryssus doryssus } \\
\text { (Swainson, 1831) }\end{array}$ & $X$ & $x$ & & & $X$ & \\
\hline 59 & Urbanus esma Evans, 1952 & & & & & $\mathrm{X}$ & \\
\hline 60 & Urbanus esta Evans, 1952 & & $\mathrm{X}$ & $x$ & & & \\
\hline 61 & Urbanus procne (Plötz, 1880) & & $\mathrm{X}$ & & & & \\
\hline 62 & Urbanus pronta Evans, 1951 & $X$ & & & & & \\
\hline 63 & $\begin{array}{l}\text { Urbanus proteus proteus } \\
\text { (Linnaeus, 1758) }\end{array}$ & & & & $\mathrm{X}$ & $\mathrm{x}$ & \\
\hline 64 & Urbanus simplicius (Stoll, 1790) & $X$ & $x$ & $\mathrm{X}$ & $X$ & $X$ & $X$ \\
\hline 65 & Urbanus teleus (Hübner, 1821) & $X$ & $x$ & $\mathrm{X}$ & & & $\mathrm{X}$ \\
\hline 66 & Urbanus velinus (Plötz, 1880) & $X$ & & & & & \\
\hline \multirow[t]{2}{*}{67} & Urbanus virescens (Mabille, 1877) & $x$ & $x$ & & & $X$ & \\
\hline & Total & 49 & 22 & 11 & 5 & 11 & 6 \\
\hline
\end{tabular}




\begin{tabular}{|c|c|c|c|c|c|c|c|}
\hline & & $\mathrm{KL}$ & UT & OU & SO & $\mathrm{CA}$ & $\mathrm{VI}$ \\
\hline & Pyrgini & & & & & & \\
\hline 68 & $\begin{array}{l}\text { Achlyodes busirus busirus } \\
\text { (Cramer, 1779) }\end{array}$ & $x$ & & $x$ & & & \\
\hline 69 & $\begin{array}{l}\text { Achlyodes mithridates thraso } \\
\text { (Hübner, [1807]) }\end{array}$ & $X$ & & $X$ & & & $x$ \\
\hline 70 & Anastrus neaeris narva Evans, 1953 & $x$ & & & & $x$ & \\
\hline 71 & $\begin{array}{l}\text { Anastrus sempiternus simplicior } \\
\text { (Möschler, 1877) }\end{array}$ & $x$ & & & & $\mathrm{X}$ & \\
\hline 72 & $\begin{array}{l}\text { Anastrus tolimus robigus } \\
\text { (Plötz, 1884) }\end{array}$ & $x$ & $x$ & & & & \\
\hline 73 & Camptopleura auxo (Möschler, 1879) & $x$ & $x$ & & & & $x$ \\
\hline 74 & $\begin{array}{l}\text { Charidia lucaria lucaria } \\
\text { (Hewitson, 1868) }\end{array}$ & $x$ & & & & & $x$ \\
\hline 75 & Chiomara mithrax (Möschler, 1879) & & $x$ & & & & \\
\hline 76 & $\begin{array}{l}\text { Cogia calchas } \\
\text { (Herrich-Schäffer, 1869) }\end{array}$ & $x$ & $x$ & $X$ & & & \\
\hline 77 & Cogia hassan hassan Butler, 1870 & $x$ & & & & & $x$ \\
\hline 78 & Cycloglypha enega (Möschler, 1877) & $X$ & & & & & \\
\hline 79 & $\begin{array}{l}\text { Cycloglypha thrasibulus thrasibulus } \\
\text { (Fabricius, 1793) }\end{array}$ & $x$ & $\mathrm{X}$ & & & $\mathrm{X}$ & \\
\hline 80 & $\begin{array}{l}\text { Cycloglypha tisias } \\
\text { (Godman \& Salvin, 1896) }\end{array}$ & $x$ & $x$ & & & & \\
\hline 81 & Cyclosemia earina (Hewitson, 1878) & $x$ & $x$ & & & & \\
\hline 82 & $\begin{array}{l}\text { Cyclosemia herennius herennius } \\
\text { (Stoll, 1782) }\end{array}$ & $x$ & $x$ & & & & \\
\hline 83 & Ebrietas elaudia livius Mabille, 1898 & $X$ & $\mathrm{X}$ & $X$ & & $x$ & $\mathrm{X}$ \\
\hline 84 & Ebrietas infanda (Butler, 1877) & $x$ & & & & & \\
\hline 85 & Eracon clinias (Mabille, 1878) & $X$ & & & & & \\
\hline 86 & Eracon paulinus (Stoll, 1782) & $x$ & $x$ & & & & \\
\hline 87 & Gesta gesta (Herrich-Schäffer, 1863) & & & & $X$ & & \\
\hline 88 & $\begin{array}{l}\text { Gindanes brebisson phagesia } \\
\text { (Hewitson, 1868) }\end{array}$ & $x$ & & & & & \\
\hline 89 & $\begin{array}{l}\text { Gorgythion begga pyralina } \\
\text { (Möschler, 877) }\end{array}$ & $x$ & $x$ & & & & \\
\hline 90 & $\begin{array}{l}\text { Gorgythion beggina escalophoides } \\
\text { Evans, } 1953\end{array}$ & & $x$ & & & & \\
\hline 91 & Gorgythion plautia (Möschler, 1877) & $x$ & $x$ & & & & \\
\hline 92 & $\begin{array}{l}\text { Helias phalaenoides phalaenoides } \\
\text { Fabricius, } 1807\end{array}$ & $x$ & $x$ & $X$ & & $x$ & \\
\hline 93 & Heliopetes alana (Reakirt, 1868) & & $x$ & & & & \\
\hline 94 & Heliopetes arsalte (Linnaeus, 1758) & $x$ & $X$ & & & & $x$ \\
\hline 95 & $\begin{array}{l}\text { Heliopetes macaira orbigera } \\
\text { (Mabille, 1888) }\end{array}$ & & $x$ & & $X$ & & \\
\hline 96 & Heliopetes petrus (Hübner, [1819]) & $x$ & $x$ & & & & \\
\hline 97 & $\begin{array}{l}\text { Iliana heros heros } \\
\text { (Mabille \& Boullet, 1917) }\end{array}$ & $x$ & & & & & \\
\hline 98 & Marela tamyris tamyris Mabille, 1903 & $x$ & & $X$ & & & \\
\hline 99 & $\begin{array}{l}\text { Marela tamyroides } \\
\text { (C. Felder \& R. Felder, 1867) }\end{array}$ & $x$ & & & & & \\
\hline 100 & $\begin{array}{l}\text { Milanion hemes hemes } \\
\text { (Cramer, 1777) }\end{array}$ & $X$ & & & & & \\
\hline 101 & Milanion sp. & $X$ & & & & & \\
\hline
\end{tabular}

\begin{tabular}{|c|c|c|c|c|c|c|c|}
\hline & & $\mathrm{KL}$ & UT & OU & SO & $\mathrm{CA}$ & $\mathrm{VI}$ \\
\hline 102 & $\begin{array}{l}\text { Morvina fissimacula rema } \\
\text { Evans, } 1953\end{array}$ & $X$ & & & & & \\
\hline 103 & Morvina morvus para Evans, 1953 & $x$ & & & & & \\
\hline 104 & Mylon cristata Austin, 2000 & $x$ & & & & & \\
\hline 105 & Nisoniades bessus (Möschler, 1877) & $x$ & $x$ & $x$ & $x$ & & $x$ \\
\hline 106 & $\begin{array}{l}\text { Nisoniades macarius } \\
\text { (Herrich-Schäffer, 1870) }\end{array}$ & $x$ & $x$ & $X$ & & $x$ & \\
\hline 107 & Nisoniades mimas (Cramer, 1775) & $x$ & $x$ & & $x$ & $x$ & \\
\hline 108 & Nisoniades nyctineme (Butler, 1877) & & $x$ & $x$ & & & $x$ \\
\hline 109 & $\begin{array}{l}\text { Nisoniades rubescens } \\
\text { (Möschler, 1877) }\end{array}$ & & $x$ & & & & \\
\hline 110 & $\begin{array}{l}\text { Ouleus fridericus fridericus } \\
\text { (Geyer, 1832) }\end{array}$ & $x$ & $x$ & $x$ & & & $x$ \\
\hline 111 & Paches gladiatus (Butler, 1870) & & $x$ & & & & \\
\hline 112 & Pachyneuria damon (Bell, 1937) & $x$ & & & & & \\
\hline 113 & $\begin{array}{l}\text { Pachyneuria duidae duidae } \\
\text { (Bell, 1932) }\end{array}$ & $x$ & $x$ & & & & \\
\hline 114 & Pachyneuria helena (Hayward, 1939) & $x$ & & $x$ & & & \\
\hline 115 & $\begin{array}{l}\text { Pachyneuria herophile } \\
\text { (Hayward, 1940) }\end{array}$ & & $x$ & & & & \\
\hline 116 & $\begin{array}{l}\text { Pachyneuria lineatopunctata belema } \\
\text { Evans, } 1953\end{array}$ & & & $X$ & & & \\
\hline 117 & $\begin{array}{l}\text { Paramimus scurra scurra } \\
\text { (Hübner, [1809]) }\end{array}$ & $x$ & & $X$ & & & \\
\hline 118 & $\begin{array}{l}\text { Pellicia dimidiata meno } \\
\text { (Mabille, 1889) }\end{array}$ & $x$ & $x$ & & & & $x$ \\
\hline 119 & Pseudodrephalys sohni Burns, 1999 & $x$ & & & & & \\
\hline 120 & Pyrgus orcus (Stoll, 1780) & $x$ & $x$ & $x$ & $x$ & & $x$ \\
\hline 121 & $\begin{array}{l}\text { Pythonides grandis assecla } \\
\text { Mabille, } 1883\end{array}$ & $x$ & & & & & \\
\hline 122 & $\begin{array}{l}\text { Pythonides herennius herennius } \\
\text { Geyer, [1838] }\end{array}$ & $x$ & & $x$ & & $x$ & \\
\hline 123 & $\begin{array}{l}\text { Pythonides jovianus fabricii } \\
\text { Kirby, } 1871\end{array}$ & $x$ & $x$ & & & $x$ & \\
\hline 124 & Pythonides lerina (Hewitson, 1868) & $x$ & $x$ & & & & \\
\hline 125 & Pythonides limaea (Hewitson, 1868) & $x$ & & & & & \\
\hline 126 & Quadrus cerialis (Stoll, 1782) & $x$ & $x$ & & & & \\
\hline 127 & $\begin{array}{l}\text { Quadrus contubernalis } \\
\text { contubernalis (Mabille, 1883) }\end{array}$ & $x$ & & & & & \\
\hline 128 & $\begin{array}{l}\text { Quadrus deyrollei portulana } \\
\text { (Plötz, 1884) }\end{array}$ & $X$ & $x$ & & & & \\
\hline 129 & Quadrus fanda Evas, 1953 & $x$ & & & & & \\
\hline 130 & $\begin{array}{l}\text { Sophista aristoteles aristoteles } \\
\text { (Westwood, 1852) }\end{array}$ & $x$ & & & & & \\
\hline 131 & Sostrata festiva (Erichson, [1849]) & $x$ & & & & $x$ & \\
\hline 132 & Spathilepia clonius (Cramer, 1775) & $x$ & $x$ & & & & \\
\hline 133 & $\begin{array}{l}\text { Staphylus corumba } \\
\text { (Williams \& Bell, 1940) }\end{array}$ & $x$ & & & & & \\
\hline 134 & $\begin{array}{l}\text { Staphylus melangon epicaste } \\
\text { Mabille, } 1903\end{array}$ & $X$ & & & & & \\
\hline 135 & $\begin{array}{l}\text { Telemiades amphion amphion } \\
\text { (Geyer, 1832) }\end{array}$ & $X$ & & $X$ & & & \\
\hline
\end{tabular}




\begin{tabular}{|c|c|c|c|c|c|c|c|}
\hline & & $\mathrm{KL}$ & UT & OU & SO & $\mathrm{CA}$ & VI \\
\hline 136 & Telemiades antiope tosca Evans, 1953 & $X$ & & & & & \\
\hline 137 & Telemiades corbulo (Stoll, 1781) & $\mathrm{X}$ & & & & & \\
\hline 138 & $\begin{array}{l}\text { Telemiades delalande } \\
\text { (Latreille, [1824]) }\end{array}$ & & $\mathrm{X}$ & & & & \\
\hline 139 & Telemiades epicalus Hübner, [1819] & $x$ & & & & & \\
\hline 140 & Telemiades penidas (Hewitson, 1867) & $X$ & $\mathrm{X}$ & & & & \\
\hline 141 & Telemiades sp. & $x$ & & & & & \\
\hline 142 & Telemiades vansa Evans, 1953 & $X$ & $X$ & & & & \\
\hline 143 & Viola violella (Mabille, 1898) & $\mathrm{X}$ & $x$ & & $\mathrm{x}$ & & \\
\hline 144 & Xenophanes tryxus (Stoll, 1780) & $X$ & $X$ & $X$ & & $x$ & $\mathrm{X}$ \\
\hline \multirow[t]{4}{*}{145} & $\begin{array}{l}\text { Zera tetrastigma tetrastigma } \\
\text { (Sepp, [1847]) }\end{array}$ & $X$ & & & & & \\
\hline & Total & 67 & 39 & 17 & 6 & 11 & 12 \\
\hline & Total (Eudamini+Pyrgini) & 116 & 61 & 28 & 11 & 22 & 18 \\
\hline & Hesperiinae & & & & & & \\
\hline 146 & Aides brino (Stoll, 1781) & $x$ & & & & & \\
\hline 147 & Aides duma argyrina Cowan, 1970 & $X$ & & & & & \\
\hline 148 & $\begin{array}{l}\text { Anatrytone barbara } \\
\text { (Williams \& Bell, 1931) }\end{array}$ & & $X$ & & & & \\
\hline 149 & Anthoptus epictetus (Fabricius, 1793) & $X$ & $X$ & $x$ & & & \\
\hline 150 & Anthoptus insignis (Plötz, 1882) & $x$ & $x$ & $x$ & & & \\
\hline 151 & Apaustus menes (Stoll, 1782) & & $x$ & & & & \\
\hline 152 & Arita arita (Schaus, 1902) & $x$ & & & & & \\
\hline 153 & Arotis kayei (Bell, 1932) & & $x$ & & & & \\
\hline 154 & Arotis pandora (Lindsey, 1925) & $X$ & & & & & \\
\hline 155 & Artines aepitus (Geyer, 1832) & $X$ & $X$ & & & & \\
\hline 156 & Callimormus alsimo (Möschler, 1883) & & $X$ & & & & \\
\hline 157 & Callimormus corades (C. Felder, 1862) & $X$ & $X$ & & & & \\
\hline 158 & $\begin{array}{l}\text { Callimormus radiola radiola } \\
\text { (Mabille, 1878) }\end{array}$ & $X$ & $X$ & & & & \\
\hline 159 & $\begin{array}{l}\text { Callimormus saturnus } \\
\text { (Herrich-Schäffer, 1869) }\end{array}$ & $X$ & & $X$ & & & \\
\hline 160 & Calpodes ethlius (Stoll, 1782) & $x$ & $X$ & & $x$ & $x$ & \\
\hline 161 & $\begin{array}{l}\text { Carystoides basoches } \\
\text { (Latreille, [1824]) }\end{array}$ & $X$ & & & & & \\
\hline 162 & $\begin{array}{l}\text { Carystoides cathaea } \\
\text { (Hewitson, 1866) }\end{array}$ & $X$ & & & & & \\
\hline 163 & $\begin{array}{l}\text { Carystoides sicania orbius } \\
\text { (Godman, 1901) }\end{array}$ & $X$ & & & & & \\
\hline 164 & $\begin{array}{l}\text { Carystus phorcus phorcus } \\
\text { (Cramer, 1777) }\end{array}$ & & $X$ & & & & \\
\hline 165 & Cobalopsis miaba (Schaus, 1902) & $X$ & $x$ & & & & \\
\hline 166 & $\begin{array}{l}\text { Cobalopsis nero } \\
\text { (Herrich-Schäffer, 1869) }\end{array}$ & $X$ & & & & & \\
\hline 167 & Cobalus calvina (Hewitson, 1866) & $X$ & & & & & \\
\hline 168 & $\begin{array}{l}\text { Cobalus virbius virbius } \\
\text { (Cramer, 1777) }\end{array}$ & $X$ & $X$ & & & & \\
\hline 169 & Conga chydaea (Butler, 1877) & $\mathrm{X}$ & & & & & \\
\hline 170 & Corticea corticea (Plötz, 1882) & $\mathrm{X}$ & $x$ & $x$ & & & \\
\hline 171 & Cymaenes alumna (Butler, 1877) & $\mathrm{X}$ & $X$ & $\mathrm{x}$ & & & $X$ \\
\hline
\end{tabular}

\begin{tabular}{|c|c|c|c|c|c|c|c|}
\hline & & $\mathrm{KL}$ & UT & OU & so & $\mathrm{CA}$ & $\mathrm{Vl}$ \\
\hline 172 & Cymaenes cavalla Evans, 1955 & & $x$ & & & & \\
\hline 173 & Cymaenes chela Evans, 1955 & & $x$ & & & & \\
\hline 174 & $\begin{array}{l}\text { Cymaenes tripunctus theogenis } \\
\text { (Capronnier, 1874) }\end{array}$ & $x$ & & $x$ & & & \\
\hline 175 & $\begin{array}{l}\text { Cynea bistrigula } \\
\text { (Herrich-Schäffer, 1869) }\end{array}$ & $x$ & & & & $x$ & \\
\hline 176 & Cynea diluta (Herrich-Schäffer, 1869) & $x$ & $x$ & & & & \\
\hline 177 & Cynea popla Evans, 1955 & $x$ & & & & & \\
\hline 178 & Cynea robba robba Evans, 1955 & $x$ & & & & & \\
\hline 179 & $\begin{array}{l}\text { Damas clavus } \\
\text { (Herrich-Schäffer, 1869) }\end{array}$ & $x$ & $x$ & & & & \\
\hline 180 & Decinea lucifer (Hübner, [1831]) & & $x$ & & & & \\
\hline 181 & Dubiella dubius (Stoll, 1781) & $x$ & & & & & \\
\hline 182 & $\begin{array}{l}\text { Dubiella fiscella fiscella } \\
\text { (Hewitson, 1877) }\end{array}$ & $x$ & & & & & \\
\hline 183 & $\begin{array}{l}\text { Ebusus ebusus ebusus } \\
\text { (Cramer, 1780) }\end{array}$ & $x$ & $x$ & & & & \\
\hline 184 & Enosis angularis (Möschler, 1877) & & & & & $x$ & \\
\hline 185 & Enosis blotta Evans, 1955 & $x$ & & & & & \\
\hline 186 & Enosis uza pruinosa (Plötz, 1882) & $x$ & & & & & \\
\hline 187 & Euphyes peneia (Godman, 1900) & $x$ & $x$ & & & & \\
\hline 188 & $\begin{array}{l}\text { Eutocus vetulus vetulus } \\
\text { (Mabille, 1883) }\end{array}$ & & $x$ & & & & \\
\hline 189 & $\begin{array}{l}\text { Eutychide subcordata subcordata } \\
\text { (Herrich-Schäffer, 1869) }\end{array}$ & $x$ & & & & & \\
\hline 190 & Flaccilla aecas (Stoll, 1781) & $x$ & & & & & \\
\hline 191 & $\begin{array}{l}\text { Hylephila phyleus phyleus } \\
\text { (Drury, 1773) }\end{array}$ & $x$ & $x$ & & $x$ & $x$ & $x$ \\
\hline 192 & $\begin{array}{l}\text { Justinia justinianus dappa } \\
\text { Evans, } 1955\end{array}$ & $x$ & $x$ & & & & \\
\hline 193 & $\begin{array}{l}\text { Justinia phaetusa phaetusa } \\
\text { (Hewitson, 1866) }\end{array}$ & & & & & $x$ & \\
\hline 194 & Lento ferrago (Plötz, 1884) & $x$ & & & & & \\
\hline 195 & $\begin{array}{l}\text { Lerema ancillaris ancillaris } \\
\text { (Butler, 1877) }\end{array}$ & & & & $x$ & & \\
\hline 196 & Methionopsis ina (Plötz, 1882) & $x$ & $x$ & & & & $x$ \\
\hline 197 & Mnaseas bicolor inca Bell, 1930 & $x$ & $X$ & & & & \\
\hline 198 & Mnasilus allubita (Butler, 1877) & & & $x$ & & & \\
\hline 199 & $\begin{array}{l}\text { Mnasitheus chrysophrys } \\
\text { (Mabille, 1891) }\end{array}$ & $x$ & & & & & \\
\hline 200 & Mnasitheus forma Evans, 1955 & $x$ & & & & & \\
\hline 201 & Molo mango (Guenée, 1865) & $x$ & & & & & $x$ \\
\hline 202 & $\begin{array}{l}\text { Monca telata telata } \\
\text { (Herrich-Schäffer, 1869) }\end{array}$ & & & $\mathrm{X}$ & & & \\
\hline 203 & Morys compta compta (Butler, 1877) & $x$ & $x$ & $x$ & & & \\
\hline 204 & Morys geisa geisa (Möschler, 1879) & $x$ & & & & & \\
\hline 205 & Nastra chao (Mabille, 1898) & & $x$ & & & & \\
\hline 206 & $\begin{array}{l}\text { Neoxeniades braesia braesia } \\
\text { (Hewitson, 1867) }\end{array}$ & $x$ & & & & & \\
\hline 207 & Neoxeniades cincia (Hewitson, 1867) & $x$ & & & & & \\
\hline 208 & Niconiades xanthaphes Hübner, [1821] & $x$ & $x$ & & & & \\
\hline
\end{tabular}




\begin{tabular}{|c|c|c|c|c|c|c|c|}
\hline & & $\mathrm{KL}$ & UT & OU & SO & CA & $\mathrm{VI}$ \\
\hline 209 & $\begin{array}{l}\text { Nyctelius nyctelius nyctelius } \\
\text { (Latreille, [1824]) }\end{array}$ & $x$ & $x$ & & & $X$ & \\
\hline 210 & $\begin{array}{l}\text { Onophas columbaria columbaria } \\
\text { (Herrich-Schäffer, 1870) }\end{array}$ & $x$ & & & & $X$ & \\
\hline 211 & Orses cynisca (Swainson, 1821) & $x$ & & & & & \\
\hline 212 & $\begin{array}{l}\text { Panoquina fusina fusina } \\
\text { (Hewitson, 1868) }\end{array}$ & $x$ & & & & & \\
\hline 213 & Panoquina hecebolus (Scudder, 1872) & $x$ & & & & & \\
\hline 214 & $\begin{array}{l}\text { Panoquina lucas lucas } \\
\text { (Fabricius, 1793) }\end{array}$ & $x$ & $x$ & $x$ & & $X$ & $x$ \\
\hline 215 & $\begin{array}{l}\text { Panoquina ocola ocola } \\
\text { (Edwards, 1863) }\end{array}$ & $x$ & $\mathrm{X}$ & & $X$ & $X$ & \\
\hline 216 & Papias ignarus (Bell, 1932) & $x$ & & & & & \\
\hline 217 & Papias phainis Godman, 1900 & $x$ & $x$ & $X$ & & & \\
\hline 218 & $\begin{array}{l}\text { Papias subcostulata } \\
\text { (Herrich-Schäffer, 1870) }\end{array}$ & $x$ & $x$ & $X$ & & & \\
\hline 219 & $\begin{array}{l}\text { Paracarystus hypargyra } \\
\text { (Herrich-Schäffer, 1869) }\end{array}$ & $x$ & & & & & \\
\hline 220 & $\begin{array}{l}\text { Paracarystus menestries rona } \\
\text { (Hewitson, 1866) }\end{array}$ & $x$ & $\mathrm{X}$ & & & $X$ & \\
\hline 221 & Parphorus sp. & $x$ & & & & & \\
\hline 222 & $\begin{array}{l}\text { Parphorus storax storax } \\
\text { (Mabille, 1891) }\end{array}$ & $x$ & & & & & \\
\hline 223 & $\begin{array}{l}\text { Perichares philetes philetes } \\
\text { (Gmelin, [1790]) }\end{array}$ & $x$ & & & & & \\
\hline 224 & Phanes aletes (Geyer, 1832) & $x$ & & $x$ & & & \\
\hline 225 & Phlebodes campo sifax Evans, 1955 & $x$ & & & & & \\
\hline 226 & Phlebodes pertinax (Stoll, 1781) & $x$ & & & & & \\
\hline 227 & Polites vibex catilina (Plötz, 1886) & $x$ & & & & & \\
\hline 228 & Pompeius amblyspila (Mabille, 1898) & & & & $\mathrm{X}$ & & \\
\hline 229 & Pompeius dares (Plötz, 1883) & & $x$ & & & & \\
\hline 230 & Pompeius pompeius (Latreille, [1824]) & $x$ & $\mathrm{X}$ & $x$ & $\mathrm{X}$ & $x$ & $x$ \\
\hline 231 & Quasimellana angra (Evans, 1955) & $x$ & & & & & \\
\hline 232 & $\begin{array}{l}\text { Quinta cannae } \\
\text { (Herrich-Schäffer, 1869) }\end{array}$ & $x$ & & & & & \\
\hline 233 & Remella remus (Fabricius, 1798) & $x$ & $\mathrm{X}$ & & & & \\
\hline 234 & Saliana chiomara (Hewitson, 1867) & $x$ & & & & & \\
\hline 235 & Saliana esperi esperi Evans, 1955 & $x$ & $\mathrm{X}$ & & & & \\
\hline 236 & Saliana fusta Evans, 1955 & $x$ & & & & $x$ & \\
\hline 237 & Saliana Iongirostris (Sepp, [1840]) & $x$ & & & & & \\
\hline 238 & Saliana morsa Evans, 1955 & $x$ & & & & & \\
\hline 239 & Saliana nigel Evans, 1955 & & & & & $x$ & \\
\hline 240 & Saliana saladin culta Evans, 1955 & $x$ & & & & & \\
\hline 241 & Saliana salius (Cramer, 1775) & $x$ & $x$ & & & & \\
\hline 242 & Saliana triangularis (Kaye, 1914) & $x$ & & $x$ & & $x$ & \\
\hline 243 & Saturnus metonidia (Schaus, 1902) & $x$ & & & & & \\
\hline 244 & $\begin{array}{l}\text { Saturnus reticulata meton } \\
\text { (Mabille, 1891) }\end{array}$ & $x$ & & & & & \\
\hline 245 & $\begin{array}{l}\text { Saturnus saturnus saturnus } \\
\text { (Fabricius, 1787) }\end{array}$ & $x$ & & & & & \\
\hline 246 & Sodalia sodalis (Butler, 1877) & $x$ & $x$ & & & & \\
\hline 247 & Synapte silius (Latreille, [1824]) & & & & & $x$ & \\
\hline
\end{tabular}

\begin{tabular}{|c|c|c|c|c|c|c|c|}
\hline & & $\mathrm{KL}$ & UT & $\mathrm{OU}$ & so & $\mathrm{CA}$ & $\mathrm{VI}$ \\
\hline 248 & Talides sinois Hübner, [1819] & $x$ & & & & & \\
\hline 249 & Thoon ponka Evans, 1955 & $x$ & & & & & \\
\hline 250 & Thracides phidon (Cramer, 1779) & $x$ & & & & & \\
\hline 251 & Turesis basta Evans, 1955 & & & & & $x$ & \\
\hline 252 & $\begin{array}{l}\text { Vacerra bonfilius litana } \\
\text { (Hewitson, 1866) }\end{array}$ & $\mathrm{X}$ & & & & & \\
\hline 253 & Vehilius celeus celeus (Mabille, 1891) & & $x$ & & & & \\
\hline 254 & Vehilius inca (Scudder, 1872 & $x$ & $x$ & $x$ & & & \\
\hline 255 & Vehilius lugubris Lindsey, 1925 & $x$ & $x$ & $x$ & & & \\
\hline 256 & $\begin{array}{l}\text { Vehilius stictomenes stictomenes } \\
\text { (Butler, 1877) }\end{array}$ & $x$ & $x$ & $x$ & $\mathrm{x}$ & & $X$ \\
\hline 257 & Vehilius vetula (Mabille, 1878) & $x$ & $x$ & $x$ & & $x$ & $x$ \\
\hline 258 & Venas caerulans (Mabille, 1878) & $x$ & $x$ & & & & \\
\hline 259 & Venas evans (Butler, 1877) & $x$ & & & & & \\
\hline 260 & $\begin{array}{l}\text { Vertica verticalis grandipuncta } \\
\text { (Mabille, 1883) }\end{array}$ & $X$ & & & & & \\
\hline 261 & Vettius fantasos (Cramer, 1780) & $x$ & & & & & \\
\hline 262 & $\begin{array}{l}\text { Vettius lafrenaye pica } \\
\text { (Herrich-Schäffer, 1869) }\end{array}$ & $\mathrm{X}$ & & & & & \\
\hline 263 & $\begin{array}{l}\text { Vettius marcus marcus } \\
\text { (Fabricius, 1787) }\end{array}$ & $x$ & & $x$ & & & \\
\hline 264 & Vettius monacha (Plötz, 1882) & $x$ & & & & & \\
\hline 265 & Vettius phyllus phyllus (Cramer, 1777) & $x$ & & & & $x$ & \\
\hline 266 & Vettius richardi (Weeks, 1906) & & $x$ & & & $x$ & \\
\hline 267 & Vettius triangularis (Hübner, [1831]) & $x$ & $x$ & & & & \\
\hline 268 & Virga virginius (Möschler, 1883) & & $x$ & $x$ & & & $x$ \\
\hline 269 & $\begin{array}{l}\text { Wallengrenia otho clavus } \\
\text { (Erichson, [1849]) }\end{array}$ & $\mathrm{X}$ & $x$ & $X$ & $X$ & & \\
\hline 270 & $\begin{array}{l}\text { Wallengrenia premnas } \\
\text { (Wallengren, 1860) }\end{array}$ & $x$ & $\mathrm{X}$ & & & & \\
\hline 271 & $\begin{array}{l}\text { Xeniades chalestra chalestra } \\
\text { (Hewitson, 1866) }\end{array}$ & & $x$ & & & & \\
\hline 272 & Zariaspes mys (Hübner, [1808]) & & & $x$ & & & \\
\hline & Total & 102 & 54 & 23 & 8 & 19 & 9 \\
\hline & Total (Hesperiidae) & 225 & 116 & 52 & 19 & 41 & 27 \\
\hline
\end{tabular}

Tabela 2 - Número de espécies de Hesperiidae reconhecidas até o momento para as oito áreas de endemismo reconhecidas da Amazônia brasileira (sensu Cracraft 1985 e Silva et al. 2002). Note que não existem dados disponíveis para três das oito áreas.

\begin{tabular}{ccc}
\hline $\begin{array}{c}\text { Riqueza } \\
(\text { spp. })\end{array}$ & $\begin{array}{c}\text { Área de } \\
\text { Endemismo }\end{array}$ & Fonte \\
\hline 272 & Belém & $\begin{array}{c}\text { (Mielke 1973; presente estudo) } \\
\text { (D'Almeida 1937; Ebert 1965; Mielke 1973; } \\
\text { Mielke e Casagrande 1991) }\end{array}$ \\
\hline 251 & Guiana & (Emmel e Austin 1990) \\
\hline 205 & Rondônia & (Mielke et al. 2011) \\
\hline 152 & Inambari & (Garwood et al. 2009) \\
\hline 117 & Tapajós & \\
& Xingú & \\
& Napo & \\
& Imeri & \\
\hline
\end{tabular}


seus resultados na forma de inventários publicados (p.ex. Santarém, Óbidos, Manaus, Jarú e Alto Juruá). Até que os dados destas coletas sejam compilado, publicados e novos registros possam ser agregados, a compreensão acerca dos limites de distribuição das espécies amazônicas e suas áreas de endemismo permanecerão desconhecidas para a ciência.

\section{AGRADECIMENTOS}

Os autores agradecem ao Sr. Pierre Jauffret, proprietário da RPPN Klagesi, que faleceu de modo trágico justamente por seu empenho em conservar um dos poucos fragmentos remanescentes de Floresta Amazônica, objeto do presente estudo. Estendemos os agradecimentos ao seu filho Jacques C. Jauffret e aos demais familiares. Os autores agradecem ao Conselho Nacional de Desenvolvimento Científico e Tecnológico (CNPq) pelo financiamento parcial deste estudo, assim como pelas bolsas de produtividade (Olaf Hermann Hendirk Mielke, Mirna Martins Casagrande) e de doutorado (Eduardo Carneiro).

\section{BIBLIOGRAFIA CITADA}

Bates, H. W. 1989. The naturalist on the river Amazonas. John Murray, London. 351 pp.

D’Almeida, R.F. 1937. Excursão scientifica aos rios Cuminá e Trombetas. Memórias do Instituto Oswaldo Cruz, 32: 235-298.

Cracraft, J. 1985. Historical biogeography and patterns of differentiation within the South American avifauna: areas of endemism. Ornithological Monographs, 36: 49-84.

Ebert, H. 1965. Uma coleção de borboletas (Lepid. Rhopal.) do Rio Amapari (território do Amapá) com anotaçôes taxonômicas sobre Rhopalocera do Brasil. Papéis Avulsos do Departamento de Zoologia, 18: 65-85.

Emmel, T.C.; Austin, G.T. 1990. The tropical rain forest butterfly fauna of Rondonia, Brasil: species diversity and conservation. Tropical Lepidoptera, 1: 1-12.

Garwood, K.; Lehman, R.; Carter, W.; Carter, G. 2009. Butterflies of Southern Amazonia. 2a ed. McAllen Publishing, Texas. 374 pp.

Gascon, C.; Bierregaard Jr, R.O.; Laurance, W.F.; Rankin-deMerona, J. 2001. Deforestation and forest fragmentation in the Amazon. p. 22-30 In: Bierregaard Jr., R. O.; Gascon, C.; Lovejoy, T. E.; Mesquita, R. (Eds.). Lessons from Amazonia: the ecology and conservation of a fragmented forest. Yale University Press, New Haven.

Lamas, G. 1981. La fauna de mariposas de la Reserva de Tambopata, Madre de Dios, Perú (Lepidoptera, Papilionoidea y Hesperioidea). Revista de la Sociedad Mexicana de Lepidopterología, 6: 23-40.
Lamas, G. 1985. Los Papilionoidea (Lepidoptera) de la zona reservada de Tambopata, Madre de Dios, Perú. I. Papilionidae, Pieridae y Nymphalidae. Revista Peruana de Entomología, 27: 59-73.

Mielke, O.H.H. 1973. Contribuição ao estudo dos Hesperiidae americanos. III Espécies coletadas em duas excursōes ao Pará e Amapá, Brasil (Lepidoptera). Acta Biológica Paranaense, 2: 17-40.

Mielke, O.H.H. 2004. Hesperiidae. p. 3-11, 25-86 In: Lamas, G. (ed.) Checklist: Part 4a. Hesperioidea - Papilionoidea. In Heppner, J. B. (ed.) Atlas of Neotropical Lepidoptera. Volume 5A. Association of Tropical Lepidoptera, Gainesville. XXXV + 439pp.

Mielke, O.H.H. 2005. Catalogue of the American Hesperioidea. Vols 1-6. Sociedade Brasileira de Zoologia, Curitiba. 1536 p. (6 vol.).

Mielke, O.H.H.; Casagrande, M. M. 1991. Lepidoptera: Papilionoidea e Hesperioidea coletados na Ilha de Maracá, Alto Alegre, Roraima, parte do projeto Maracá, com uma lista complementar de Hesperiidae de Roraima. Acta Amazônica, 21: $175-210$.

Mielke, O.H.H.; Carneiro, E.; Casagrande, M. M. 2011 Borboletas (Lepidoptera: Papilionoidea e Hesperioidea) do Parque Estadual do Chandless e arredores, Acre, Brasil. Biota Neotropica, 10: 1-15.

Robbins, R.K.; Lamas, G.; Mielke, O.H.H.; Harvey, D.J.; Casagrande, M.M. 1996. Taxonomic composition and ecological structure of the species-rich butterfly community at Pakitza, Parque Nacional del Manu, Perú. p. 217-252. In: Wilson, D.E.; Sandoval, A. (Eds). Manu. The Biodiversity of Southeastern Peru. La Biodiversidad del Sureste del Perú. Lima, Editorial Horizonte.

Santos, E.C.; Mielke, O.H.H.; Casagrande, M.M. 2008. Inventários de borboletas no Brasil: estado da arte e modelo de áreas prioritárias para pesquisa com vistas à conservaçáo. Natureza \& Conservação, 6: 68-90.

Silva, J.M.C.; Novaes, F.C.; Oren, D.C. 2002. Differentiation of Xiphocolaptes (Dendrocolaptidae) across the river Xingu, Brazilian Amazonia: recognition of a new phylogenetic species and biogeographic implications. Bulletin of the British Ornithologists' Club, 122: 185-194.

Silva, J.M. C.; Rylands, A.B.; Fonseca, G.A.B. 2005. O destino das áreas de endemismo da Amazôni a. Megadiversidade, 1: 124-131.

Stichel, H. 1923. Beiträge zur Kenntnis der Riodinidenfauna Südamerikas. IV. Nord-Brasilien (Grão-Para). Zeitschrift für wissenschaftliche Insektenbiologie, 18: 268-283, 305-316.

Wallace, A.R. 1853. A narrative of travels on the Amazon and Rio Negro, with an account of the native tribes and observations on the climate, geology, and natural history of the Amazon valley. Reeve \& Co., London. 541 pp.

Recebido em 13/02/2011

Aceito em 01/06/2011 
